\title{
Awareness of preanalytical variables affecting blood glucose testing
}

\begin{abstract}
In the era of evidence based medicine laboratories play a pivotal role. Maintaining quality in laboratory results, keeping errors minimum is a daunting task. The preanalytical phase of testing is most error prone as it involves many personnel. Measures to reduce preanalytical errors include- continuous education \& training, written policies, implementation of standard operating procedures, establishment of quality indicators and communication. It is the most needed requirement world over so as to deliver accurate, precise and correct results that eventually become the foundation of diagnosis and ultimately patient care.
\end{abstract}

Keywords: pre analytical variables, diabetes mellitus
Volume 5 Issue 3 - 2018

\author{
Rateesh Sareen,' Menka Kapil,' Akanksha \\ Dutt $^{2}$ \\ 'Consultant Pathology, Santokba Durlabhji Memorial Hospital \& \\ Research Center, India \\ ${ }^{2}$ Consultant Anesthesiology, RCH \&Khandaka Hospital, India
}

Correspondence: Rateesh Sareen, Department of Pathology, Santokba Durlabhji Memorial Hospital, Bhawani Singh Road, Jaipur, India, Tel 941421647I,

Email drrateeshsareen@yahoo.co.in

\section{Introduction}

Diabetes mellitus (DM) is a common disorder affecting individuals of all ages. ${ }^{1}$ The literature study points out that there is deficiency in testing for glucose related to preanalytical and analytical errors resulting in poor determination of blood glucose levels in patients. ${ }^{2}$ Therefore, the glucose estimation in hospitals is of paramount importance in diagnosis and follows up in diabetes patients.

\section{Point of care testing (POCT)}

POCT can be done by using any of the three biochemical reactionsGlucose oxidase, Glucose dehdrogenase and Hexokinase respectively. These methods require a drop of blood from finger prick sample. In these methods the RBC lysis does not take place. As the water content of circulating cell is lower than whole plasma, these methods tend to estimate higher plasma glucose values in comparison to whole blood glucose estimation for samples with normal Hematocrit. ${ }^{3}$ The HemoCue method (HemoCue, Mission Viejo, CA) is one of the only POCT glucose techniques that lyse RBC using Saponin as the lysing agent. As the POCT device is calibrated to plasma glucose rather than blood glucose it is imperative to know the calibration by manufacturer prior to use in laboratory. ${ }^{4}$ As per the FDA data on blood glucose monitors the most frequent errors associated with POCT devices were inadequate instrument cleaning, incorrect quality control, improper technique of sampling and incorrect calibration of glucose monitor and test strip calibration by manufacturer. ${ }^{5}$ In addition, there are many drugs that interfere with glucose oxidase method. ${ }^{6}$ The POCT devices that are affected by extreme Hematocrit will give falsely elevated plasma glucose values in Polycythemia cases. As a rule of thumb for every $10 \%$ change in Hematocrit, there is a change in blood glucose in opposite direction of magnitude of the order $3.6 \mathrm{mg} / \mathrm{dl} .{ }^{7}$ Care has to be taken while measuring glucose levels in newborns with POCT devices as they have high Hematocrit and frequently have hypoglycemia. Patient with altered po $2^{8,9}$ will have unreliable glucose estimation by glucose oxidase method. Among the post analytical factors the interfacing of POCT devices with laboratory information system (LIS), which unfortunately is not done, possess a major problem with data management. ${ }^{10}$

\section{Hospital \& laboratory testing}

Pre analytical variables like patient preparation, type of sample, fasting requirement, sample storage and transport all affect laboratory test result. The main factor is the ex vivo glycolysis which should be minimal. It is prevented by placing the specimen in ice but due to technical difficulties nowadays Sodium Fluoride tubes are used for collection. ${ }^{11}$ The practice of 12 hour fasting is to cover the requirement for lipid profile testing which essentially requires 12 hour of fasting where as for fasting glucose the requirement is of 8 hours only but since the two tests are often ordered together the fasting time for glucose is exaggerated. The clinical \& laboratory institute (CLSI) $\mathrm{H} 21$ guideline also does not mention patient preparation in terms of fasting requirement. The guideline mentions that appropriate time \& diet restrictions apply to certain tests but they do not specify exactly about requirement of dietary restriction on fasting patients. ${ }^{12}$

It is the responsibility of all stakeholders who are involved in the testing process requesting physician, nurse, laboratory technician, phlebotomist to give appropriate time and maintain proper communication with the patient as these two were the confounding factors requiring major improvement as suggested by other studies in literature. ${ }^{13}$ Laboratories in particular the one which are compliant with ISO1518918, JCI maintain clear understandable written instructions for display to patients at laboratory sample collection area. ${ }^{14}$ Endogenous factors like diurnal variation ${ }^{15}$ can affect glucose measurement as the glucose values during an oral glucose tolerance test tend to be higher when test is performed in afternoon than when the test is performed in the morning. The consumption of ethanol can cause short term effect within first 2 to 4 hours of consumption including lowering of blood glucose level. ${ }^{16}$ It is quite often seen that exceptionally high glucose values are obtained by collecting blood from the arm in which an infusion of glucose is administered.

\section{Acknowledgements}

None. 


\section{Sources of funding}

None.

\section{Conflict of interest}

Author declares there is no conflict of interest.

\section{References}

1. Lefebvre P. Diabetes yesterday, today and tomorrow. The action of the International Diabetes Federation. Rev Med Liege. 2005;60(5-6):273-277.

2. Frankum S, Ogden J. Estimation of blood glucose levels by people with diabetes: A cross-sectional study. Br J Gen Pract. 2005;55(521):944-948.

3. Sacks DM. Carbohydrates. In: Ashwood ER, editor. Tietz Textbook of Clinical Chemistry. $3^{\text {rd }}$ edn. Saunders: Philadelphia; 1999. p. 750-808.

4. Turner APF, Chen B, Piletsky SA. In vitro diagnostics in diabetes: meeting the challenge. Clin Chem. 1999;45(9):1596-1601.

5. Hazard. Errors in using blood glucose monitors in hospitals. Health Devices. 1996;25(4):147-148.

6. Young DS. Effects of Drugs on Clinical Laboratory Tests. $4^{\text {th }}$ edn. AACC Press: Washington DC; 1995. p. 374-391.

7. Barreau PB, Buttery JE. The effect of haematocrit on the determination of glucose levels by reagent strip methods. MedJ Aust. 1987;147(6):286-288.

8. Kurahashi K, Maruta H, Usuda Y, et al. Influence of blood sample oxygen tension on blood glucose concentration using an enzyme-electrode method. Crit Care Med. 1997;25(2):231-235
9. Kost GJ, Vu HT, Lee JH, et al. Multicenter study on oxygen-insensitive handheld glucose point-of-care testing in critical care/hospital/ambulatory patients in the United States and Canada. Crit Care Med. 1998;26(3):581590 .

10. Main RI, Wright J, Kiechle FL. Data management programs for bedside glucose testing. Lab Med. 1994; 25(12):784-789.

11. Shi RZ, Seeley ES, Bowen R, et al. Rapid blood separation is superior to fluoride for preventing in vitro reductions in measured blood glucose concentration. J Clin Pathol. 2009;62(8):752-753.

12. Simundic AM, Bilic Zulle L, Nikolac N, et al. The quality of the extraanalytical phase of laboratory practice in some developing European countries and Mexico - a multicentric study. Clin Chem Lab Med. 2011;49(2):215-228.

13. Clinical Laboratory Standards Institute. Collection, transport, and processing of blood specimens for testing plasma-based coagulation assays: approved guideline $-6^{\text {th }}$ edn. Clinical and Laboratory Standards Institute: Wayne: 2008

14. GulderenYanikkaya Demirel. ISO 15189 accreditation: Requirements for quality and competence of medical laboratories, experience of a laboratory II. Clinical biochemistry, 2009; 42(4-5):279-283.

15. Young DS. Effects of Preanalytical Variables on Clinical Laboratory Tests. $2^{\text {nd }}$ edn. AACC Press: Washington DC; 1997.

16. Guder WG, Narayanan S, Wisser H, et al. Samples: From the Patient to the Laboratory: The Impact of Preanalytical Variables on the Quality of Laboratory Results. GIT Verlag: Darmstadt; 1996. p. 1-149. 